\title{
Functional sex differences in human primary auditory cortex
}

\author{
Liesbet Ruytjens • Janniko R. Georgiadis • Gert Holstege • \\ Hero P. Wit • Frans W. J. Albers • Antoon T. M. Willemsen
}

Received: 28 February 2007 / Accepted: 22 June 2007 / Published online: 17 August 2007

(C) Springer-Verlag 2007

\begin{abstract}
Background We used PET to study cortical activation during auditory stimulation and found sex differences in the human primary auditory cortex (PAC). Regional cerebral blood flow $(\mathrm{rCBF})$ was measured in 10 male and 10 female volunteers while listening to sounds (music or white noise) and during a baseline (no auditory stimulation).

Results and discussion We found a sex difference in activation of the left and right PAC when comparing music to noise. The PAC was more activated by music than by noise in both men and women. But this difference between the two stimuli was significantly higher in men than in women. To investigate whether this difference could be
\end{abstract}

L. Ruytjens $\cdot$ H. P. Wit

Department of Otorhinolaryngology, University Medical Center Groningen,

9700 RB Groningen, The Netherlands

\section{J. R. Georgiadis}

Department of Anatomy and Embryology,

University of Groningen, University Medical Center Groningen,

9700 AD Groningen, The Netherlands

\author{
A. T. M. Willemsen \\ Department of Nuclear Medicine and Molecular Imaging, \\ University Medical Center Groningen, \\ 9700 RB Groningen, The Netherlands \\ L. Ruytjens $(\bowtie) \cdot F$. W. J. Albers \\ Department Otorhinolaryngology, \\ University Medical Center Utrecht, \\ P.O. Box 85500, 3508 GA Utrecht, The Netherlands \\ e-mail: 1.ruytjens@umcutrecht.nl

\section{G. Holstege} \\ Center for Uroneurology, \\ University of Groningen, University Medical Center Groningen, \\ 9713 AV Groningen, The Netherlands
}

attributed to either music or noise, we compared both stimuli with the baseline and revealed that noise gave a significantly higher activation in the female PAC than in the male PAC. Moreover, the male group showed a deactivation in the right prefrontal cortex when comparing noise to the baseline, which was not present in the female group. Interestingly, the auditory and prefrontal regions are anatomically and functionally linked and the prefrontal cortex is known to be engaged in auditory tasks that involve sustained or selective auditory attention. Thus we hypothesize that differences in attention result in a different deactivation of the right prefrontal cortex, which in turn modulates the activation of the PAC and thus explains the sex differences found in the activation of the PAC.

Conclusion Our results suggest that sex is an important factor in auditory brain studies.

Keywords Brain $\cdot$ Gender $\cdot$ Hearing $\cdot$ Neuroimaging $\cdot$ PET

\section{Introduction}

Previously, investigators tried to relate sex differences in behaviour and cognition to differences in brain anatomy, function, or connectivity. A well-documented example of sex differences in the brain is the difference in overall brain size. We now know that the size of the male brain is on average $8-10 \%$ larger than the female brain [1-3]. It is also thought that, compared to the female brain, there is more hemispheric asymmetry in the anatomy of the male brain [4-7], which is supported by the finding that in the male brain, functions are represented more unilaterally [8-12].

Sexual dimorphism has also been reported for the anatomy and function of the auditory cortex. The human auditory cortex is located in the superior temporal lobe. The 

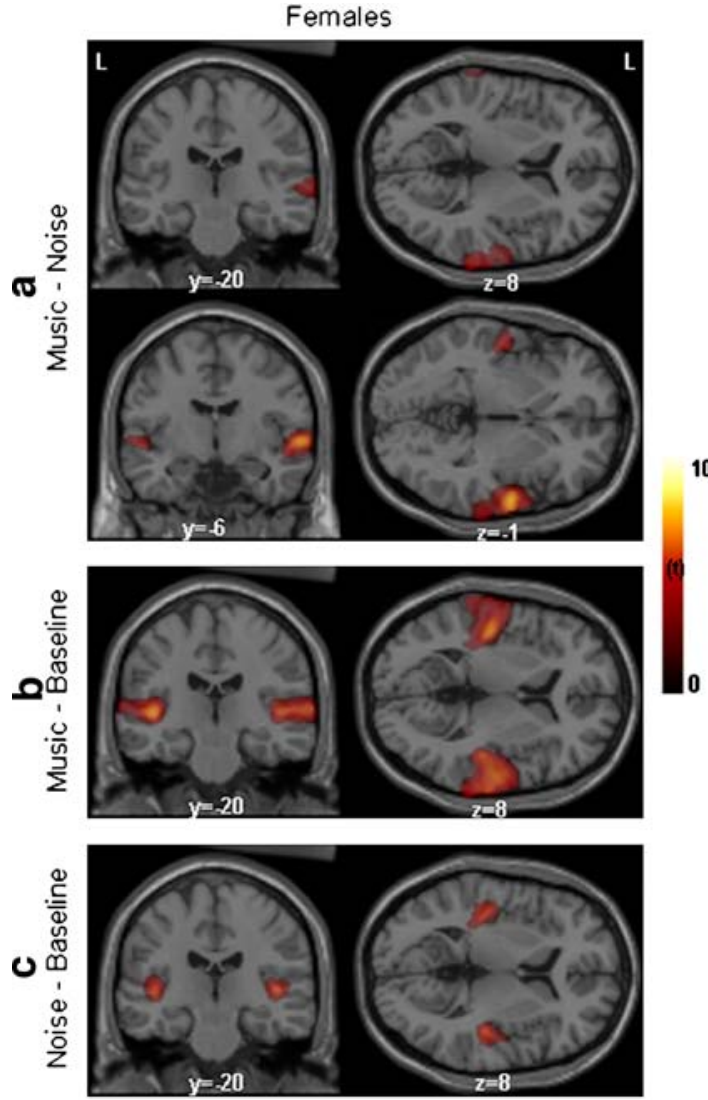
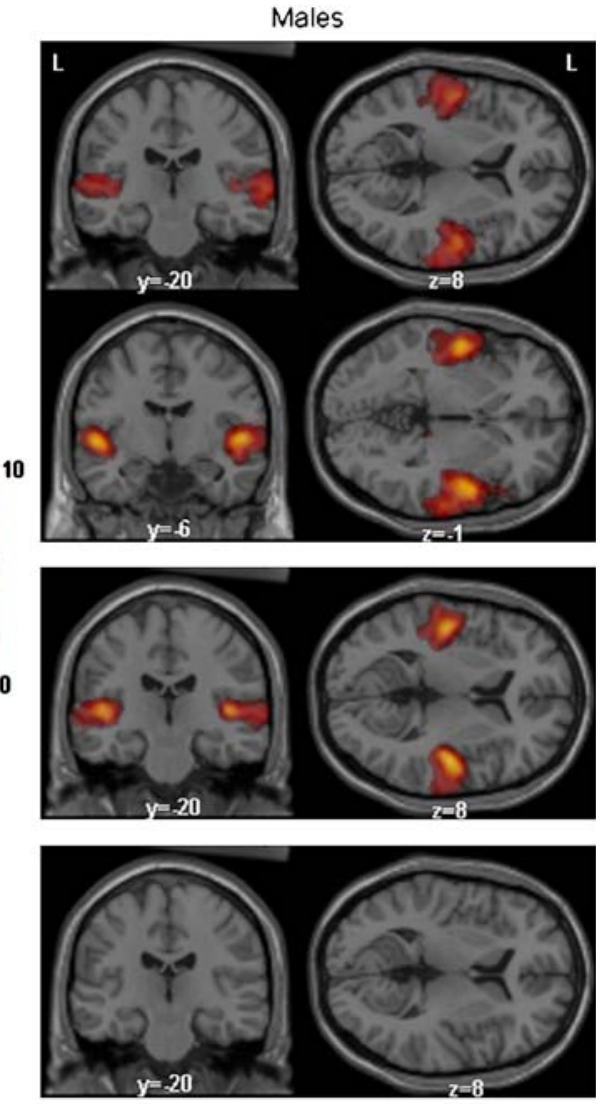

Significance threshold $p<005$ FDR corrected for multiple comparisons

Fig. 1 Spatial distribution of significant increases in brain activation in men and women when comparing the auditory processing of noise, music, and a baseline. Activations are superimposed on an anatomical MRI template of SPM2. Clusters are significant at $p<0.05$ FDR corrected for multiple comparisons. $L=$ left hemisphere, $y=-20$ and $y=-6$ means a coronal plane, respectively, $20 \mathrm{~mm}$ and $6 \mathrm{~mm}$ posterior to the anterior commissure, $z=8$ means a horizontal plane $8 \mathrm{~mm}$ dorsal to the anterior commissure, $z=-1$ means a horizontal plane $1 \mathrm{~mm}$ ventral to the

primary auditory cortex (PAC) is situated in the medial twothirds of the transverse temporal gyrus, also called Heschl's gyrus (Fig. 1). It is surrounded by secondary and associative auditory regions, which cover the lateral part of the transverse temporal gyrus and extend to the superior temporal plane. The primary auditory region was designated as area 41 by Brodmann [13], the secondary auditory regions are areas 42 and 22 [14]. An example of sexual dimorphism in the anatomy of the auditory system is in the study by Rademacher et al. [15] who reported that both left and right primary auditory cortices are larger in females than in males, although an earlier study found no differences [7]. Anatomical reports also show larger and more symmetrical auditory association cortices in females than in males $[7,16]$. However, many studies emphasize that there is intersubject variability regarding the size and location of the primary and secondary auditory cortex [17-21]. anterior commissure. a Contrasting music with noise, women showed activation in the secondary auditory areas only, whereas men showed activation in both PAC and secondary auditory areas. b Comparing music to the baseline, both women and men showed bilateral activation in the PAC and secondary auditory areas. c Comparing noise to the baseline, women showed bilateral activation in the PAC. In men, on the other hand, no significant activation was found. The differences between men and women in $\mathbf{a}$ and $\mathbf{c}$ are significant (see Table 2)

Functional sex differences in auditory processing are widely studied in the light of language. Females depend less on their left hemisphere for language processing than males in some studies [22], whereas in other studies the opposite was found $[23,24]$ or no sex difference could be detected $[25,26]$. Whether a sex difference in language processing can be detected might depend on the nature of the task [10].

Sex-based influence on activation patterns in auditory regions is also found when manipulating the amount of background noise [27], and auditory working memory tasks induce different activation patterns in males and females [28]. These studies suggest that combining men and women in auditory neuroimaging studies may obscure or bias results.

However, most of these studies focus mainly on functional sex differences in higher order (associative) brain areas, thereby ignoring possible sex differences in 
primary auditory cortices. Previously, a sex difference in activation of the PAC during lipreading was found [29]. However, it has not been investigated whether nonspeech sounds can induce different activity in the PAC in both sexes.

In the present study, we investigated potential sex differences in the activity of the primary auditory region, using different auditory stimuli.

\section{Material and methods}

\section{Subjects}

Twenty healthy, right-handed volunteers participated in this study (10 males, 10 females). The mean age for men was 23 years (range 20-25) and for women 22 years (range 1927). All volunteers gave written informed consent according to the Declaration of Helsinki and the Medical Ethics Committee of the University Medical Center Groningen approved the study. None of the subjects had any history of psychiatric or neurological disorders. Prior to the scanning, subjects were tested for their hearing thresholds using standard audiometric measures. All subjects had normal hearing thresholds $(<20 \mathrm{dBHL}, 0.25-8 \mathrm{kHz})$, and only small intersubject variations in hearing thresholds were observed.

\section{Data acquisition}

Regional cerebral blood flow (rCBF) was measured using radioactive water $\left(\left[{ }^{15} \mathrm{O}\right]\right.$-water, half-life $\left.122 \mathrm{~s}\right)$ as a tracer. A Siemens Ecat Exact HR+PET scanner, operated in threedimensional mode with a $15.5 \mathrm{~cm}$ axial field of view, acquired 63 slices simultaneously. Each subject was scanned 12 times to measure the distribution of $\left[{ }^{15} \mathrm{O}\right]$-water with a 10 minute interval between two scans to allow for decay. Each scan was performed after an intravenous bolus injection of $500 \mathrm{MBq}$ of $\left[{ }^{15} \mathrm{O}\right]$-water per scan. Except for the first scan, scanning started $30 \mathrm{~s}$ prior to injection, to account for background activation. Scanning continued for 120 seconds. The activity measured during this period was summed and used as a measure of rCBF. A scan specific calculated attenuation correction was performed to minimize interscan displacement-induced variance [30]. All subjects were scanned at fixed times on fixed weekdays, and male and female subjects were addressed to the scan dates randomly.

\section{Experimental design}

Three conditions were used in this study: baseline (no auditory stimulation), white noise (at an intensity level of
75 dBSL), and music (music of the movie "The Piano" at 75 dBSL). Both music and noise have a wide frequency range, stimulating a large number of haircells in the cochlea and hence a large portion of the cortical auditory areas, but noise has a continuous and uniform frequency spectrum whereas music is a dynamic stimulus. Each condition was presented four times in a random order. For stimulus presentation we used a clinical audiometer (Interacoustics, model AC30), a Tandberg Educational tape recorder, and E. A.RTone $3 \mathrm{~A}$ insert phones (with E.A.Rlink eartips), which have a flat frequency response between 100 and $4000 \mathrm{~Hz}$, measured in a Zwislocki-coupler. Stimuli were presented binaurally. Ten seconds before injection of radioactive water, the stimulus was started. Because the tracer reaches the brain approximately 10 seconds after injection, subjects were exposed to the stimulus for 20 seconds before the distribution of $\left[{ }^{15} \mathrm{O}\right]$-water in the brain starts. Subjects were instructed to close their eyes, not to move during the scans, and to listen to the auditory stimuli. Before each scan we informed the volunteers that the scan was about to start. Immediately after each scan the volunteers were questioned about the scan (did they hear the stimulus? were they uncomfortable or distracted?). During scanning we monitored the subjects with infrared cameras.

\section{Data analysis}

The 2002 version of Statistical Parametric Mapping (SPM2: software from the Wellcome Department of Cognitive Neurology, London, UK) was used for spatial transformations (realignment, transformation into standard stereotactic space, and smoothing with an isotropic Gaussian kernel of $8 \mathrm{~mm}$ FWHM), and statistical analysis [31]. An ANOVA estimated the following parameters: two groups (male and female), three conditions (baseline, noise, and music) and the mean perfusion to normalize for global flow differences (multigroup, conditions, and covariates). Each scan was scaled to a mean global activity of $50 \mathrm{ml} / 100 \mathrm{ml} / \mathrm{min}$. Hypotheses about regionally specific condition effects were tested to compare the estimates by using linear compounds or contrasts. The resulting set of voxel values for these contrasts constituted the associated SPM of the t-statistics.

The significance threshold used for the analysis of the two groups separately (male-female) was $p<0.05$ (false discovery rate (FDR) corrected for multiple comparisons [32]) with an extent cluster threshold of more than 8 voxels. We used AMIDE software (http://amide.sourceforge.net/) for colour scaling and display of the results on the anatomical MRI template of SPM2. For maximum statistical sensitivity and for testing the significance of the sex related differences, we conducted a region of interest (ROI) analysis in our a priori hypothesized areas, i.e. the left and right PAC, using the SPM anatomy toolbox [33] and the 
MarsBaR toolbox (MARSeille Boîte À Région d'intérêt [34]). Specifically, we created anatomical ROIs based on the three-dimensional probabilistic cytoarchitectonic maps from the SPM anatomy toolbox brains [18, 20, 33]. To compensate for differences in stereotactic space between SPM and the anatomy toolbox, a linear transformation was applied to the anatomical ROIs. MarsBaR was then used to conduct the statistical analyses on these ROIs. The statistical procedure in MarsBaR is the same as in SPM, but instead of analysing on a voxel-by-voxel basis like SPM does, all voxels in a region are averaged, and hence inferences about the whole region can be made. Also in MarsBaR, contrasts were considered significant at $p<0.05$.

\section{Results}

\section{Music versus noise}

For this contrast, in females the SPM-analysis resulted in significant bilateral activation clusters with a maximal significant voxel in the secondary auditory areas. In men much larger bilateral clusters were found covering not only the secondary auditory areas but also the PAC (Fig. 1a and Table 1). This indicates that males have a much larger activation in the PAC during music than during noise. The voxel-wise analysis of SPM did not reveal any significant activation differences between the two sexes.
The region of interest analysis in the PAC showed that females do have a larger activation in the PAC during music than during noise ( $p$ values 0.005 and 0.001 for the left and right PAC, respectively). But the difference between music and noise is much smaller than in males in both the left and right PAC ( $p$ values 0.016 and 0.008 , respectively, Table 2, Fig. 2). No significant deactivations were found (i.e. noise versus music).

To determine whether this sex difference can be attributed to the processing of either music or noise, we compared these two stimuli with a baseline without experimental auditory stimulation.

\section{Music versus baseline}

For this contrast, the SPM-analysis showed large comparable activation clusters covering primary and secondary areas in both males and females (Fig. $1 \mathrm{~b}$ and Table 1). The ROI analysis showed that there were no significant differences in the activation of the PAC between both sexes for this contrast (Table 2, Fig. 2). Again, no significant deactivations were found (baseline versus music).

\section{Noise versus baseline}

Comparing noise to the baseline, the SPM-analysis showed two significant bilateral activation clusters with maxima in the PAC for the female group. In contrast, no significant

Table 1 Overview of brain areas with statistically significant cerebral blood flow changes

\begin{tabular}{|c|c|c|c|c|c|c|c|c|}
\hline & & \multirow[t]{2}{*}{ Side } & \multirow[t]{2}{*}{ Region } & \multicolumn{3}{|c|}{ Talairach coordinates } & \multirow[t]{2}{*}{ Number of voxels } & \multirow[t]{2}{*}{ t-value } \\
\hline & & & & $\mathrm{x}$ & $\mathrm{y}$ & $\mathrm{z}$ & & \\
\hline \multirow[t]{4}{*}{ Music vs noise } & \multirow[t]{2}{*}{ Females } & Right & BA 22 & 61 & -6 & -1 & 1,459 & 7.77 \\
\hline & & Left & BA 22 & -51 & -10 & -1 & 310 & 5.02 \\
\hline & \multirow[t]{2}{*}{ Males } & Right & BA $41,42,22$ & 52 & 2 & -3 & 3,234 & 7.87 \\
\hline & & Left & BA $41,42,22$ & -51 & -8 & 0 & 2,190 & 7.87 \\
\hline \multirow[t]{4}{*}{ Music vs baseline } & \multirow[t]{2}{*}{ Females } & Right & BA $41,42,22$ & 57 & -4 & -1 & 2,750 & 8.85 \\
\hline & & Left & BA $41,42,22$ & -48 & -17 & 3 & 1,866 & 7.97 \\
\hline & \multirow[t]{2}{*}{ Males } & Right & BA $41,42,22$ & 51 & -10 & 2 & 2,475 & 10.36 \\
\hline & & Left & BA $41,42,22$ & -48 & -12 & 1 & 2,306 & 8.67 \\
\hline \multirow[t]{3}{*}{ Noise vs baseline } & \multirow[t]{2}{*}{ Females } & Right & BA 41 & 46 & -21 & 5 & 446 & 6.49 \\
\hline & & Left & BA 41 & -40 & -23 & 5 & 463 & 6.24 \\
\hline & Males & No suprathreshold clusters & & & & & & \\
\hline \multirow[t]{2}{*}{ Baseline vs noise } & Females & No suprathreshold clusters & & & & & & \\
\hline & Males & Right & BA 9 & 20 & 50 & 29 & 968 & 5.20 \\
\hline \multirow[t]{2}{*}{ Baseline vs music } & Males & No suprathreshold clusters & & & & & & \\
\hline & Females & No suprathreshold clusters & & & & & & \\
\hline
\end{tabular}

The region, covered by the whole cluster and noted in Brodmann areas, and the number of voxels in the cluster are described [14]. BA 41 corresponds to the PAC, BA 22 and 42 correspond to secondary auditory areas. Only the stereotaxic (Talairach) coordinates and t-value of the maximum of the cluster are given. The significance threshold was $p<0.05$ FDR-corrected for multiple comparisons; extent threshold was 8 voxels; voxel size was $2 \times 2 \times 2 \mathrm{~mm}$. Brain regions were identified using the Talairach atlas and the stereotactic atlas of the human brain of Mai et al. $[52,53]$ 
Table 2 Region of interest analysis of the left and right primary auditory cortex (PAC)

\begin{tabular}{lll}
\hline & \multicolumn{2}{l}{ P-value } \\
\cline { 2 - 3 } & Left PAC & Right PAC \\
\hline Music vs noise females & $0.005^{*}$ & $0.001^{*}$ \\
Males & $<0.001^{*}$ & $<0.001^{*}$ \\
Females minus males & 0.984 & 0.992 \\
Males minus females & $0.016^{*}$ & $0.008^{*}$ \\
Music vs baseline females & $<0.001^{*}$ & $<0.001^{*}$ \\
Males & $<0.001^{*}$ & $<0.001^{*}$ \\
Females minus males & 0.721 & 0.779 \\
Males minus females & 0.279 & 0.221 \\
Noise vs baseline females & $<0.001^{*}$ & $<0.001^{*}$ \\
Males & $0.016^{*}$ & $0.005^{*}$ \\
Females minus males & $0.042^{*}$ & $0.034^{*}$ \\
Males minus females & 0.958 & 0.966 \\
\hline
\end{tabular}

One-sided statistical significance for various contrasts was tested for men and women separately as well as for the differences between both. *Significant at $p<0.05$

activations were found in the male brain at a corrected level of $p<0.05$ (Fig. 1c and Table 1). Only when the data were analysed at an uncorrected threshold of $p<0.01$, a small activation appeared in the PAC of males, primarily on the right side (Fig. 3). The ROI-analysis confirmed in men the involvement of the PAC while processing noise, showing significant bilateral increases of blood flow, but these activations were significantly less than in women in the left (0.042) and right PAC ( $p=0.034)$ (Table 2).

The voxel-wise analysis of SPM also revealed a significant deactivation in the male group. This deactivation was located in the right dorsolateral part of the prefrontal cortex extending to the posterior part of the middle frontal gyrus, covering primarily BA 9 (Fig. 4 and Table 1). In contrast, no significant deactivation was found in the female group.

\section{Discussion}

Our data demonstrate a sex difference in regional cerebral blood flow in the left and right primary auditory cortex (PAC) when comparing auditory processing of music and noise. The PAC was more activated by music than by noise in both men and women. But this difference between the two stimuli was significantly higher in men than in women. To determine whether this sex difference can be attributed to the processing of music, noise, or both, we compared the two stimuli with a baseline of no auditory stimulation. Comparing music with the baseline resulted in extensive activation of the primary and secondary auditory cortex in both sexes, but no significant sex difference was found. On the other hand, a sex difference was detected in the processing of noise, because females activated their PAC significantly more than males. The finding that sex differences in auditory processing can already be detected at the level of the primary cortex is very important, because it is often assumed that sex differences act exclusively upon higher-order cortical areas.

There appears to be a mismatch between the SPM and ROI analyses because there is a difference in power between the two analysing methods. The ROI analysis pools the data of all voxels in the PAC and gives a reduced standard error, resulting in a higher t-value and hence more power [34]. Although the SPM analysis showed no activation at all for the male group and a very significant activation for the female group, it did not have enough power to detect a significant difference between the two groups. Because of the enhanced sensitivity of an ROI analysis, this analysis did show a significant sex difference. Of course, caution has to be made when pooling data to improve the sensitivity and is therefore only allowed in a priori defined regions, in this case the PAC.

The question is whether the sex difference found is caused by the experimental conditions or by other factors like anatomical differences or methodological errors. The reported differences in activation of the PAC stand or fall by a correct identification of this area in the region of interest analysis. Several authors reported substantial intersubject variability in size and location of the PAC [17-21]. To overcome this problem of variability in size and location of the PAC, we employed linear resizing and shearing as well as nonlinear warping of the brain to normalize the individual brains into a standardized stereotactic frame. In addition, a Gaussian smoothing filter of

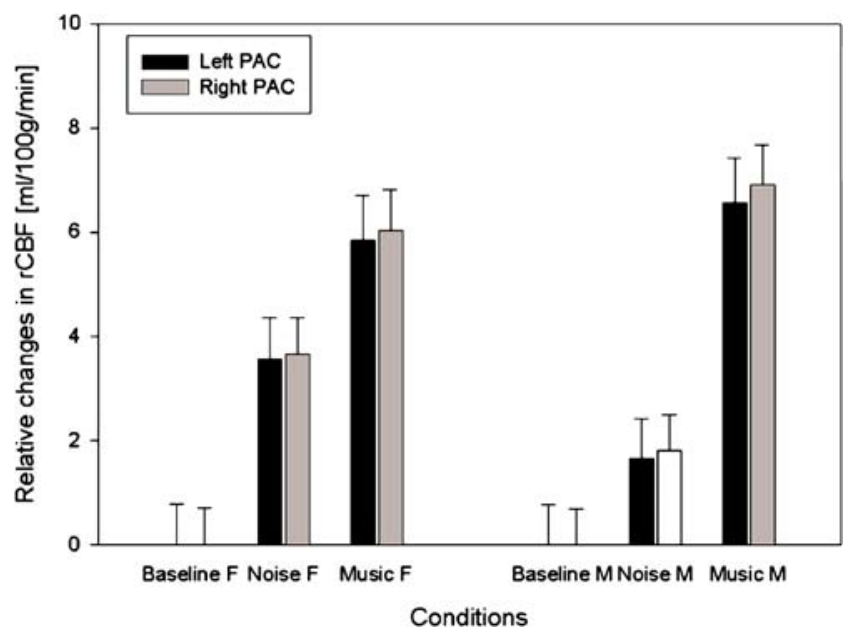

Fig. 2 Regional cerebral blood flow relative to the baseline of each group, based on all voxels in the left and right PAC (with a global mean flow of $50 \mathrm{ml} / 100 \mathrm{~g} / \mathrm{min}$ ). Error bars indicate the $90 \%$ confidence interval of the mean across subjects per condition; the confidence interval of the baseline is also given 


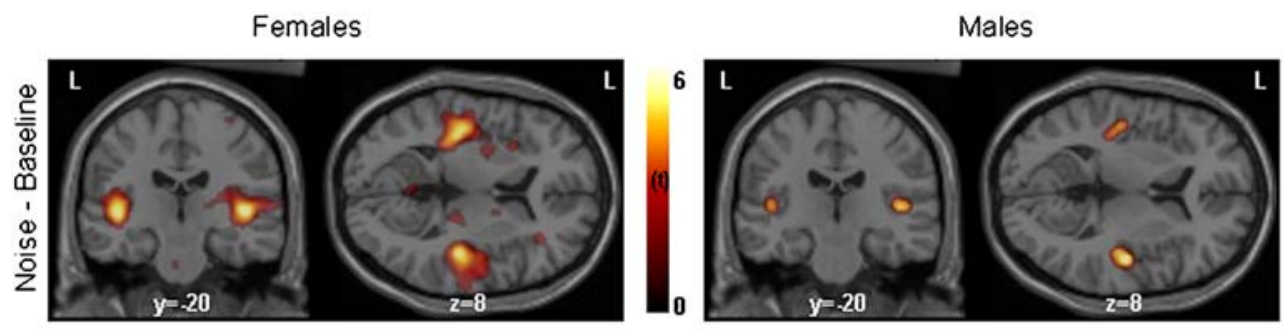

Significance threshold $p<0.01$ uncorrected for multiple comparisons

Fig. 3 Spatial distribution of significant increases in brain activation in men and women for the comparison of noise to the baseline. Clusters are significant at $p<0.01$, uncorrected for multiple comparisons. $L=\mathrm{left}$ hemisphere, $y=-20$ means a coronal plane $20 \mathrm{~mm}$ posterior to the anterior commissure, $z=8$ means a horizontal plane $8 \mathrm{~mm}$ dorsal to the anterior commissure. In contrast to Fig. 1c, at an uncorrected level men do show activation in PAC, but it is much smaller than in the female group
$8 \mathrm{~mm}$ was used to remove residual variance in brain structure that remains after the stereotactic normalization. Furthermore, we used probability maps, based on the cytoarchitecture of 10 subjects, to identify the PAC [18, $20,33]$. Other studies suggested that the volume of the PAC is bilaterally larger in females than in males [15]. It is therefore necessary that the region of interest depicts the PAC of both men and women. This is the case, because the probability maps of the SPM anatomy toolbox are based on the cytoarchitecture of five male and five female brains [18, $20,33]$. For these reasons we believe that possible intersubject or intergroup differences in size and location of the PAC do not bias our results.

If the reported sex and contrast dependent differences in rCBF patterns are not caused by underlying anatomical differences, metabolic differences, or methodological errors, they must have been induced by the experimental stimulus. During the baseline condition, subjects had to lie quietly in the scanner and no auditory stimulus was applied. Without auditory stimulation and a specific task, the variance in $\mathrm{rCBF}$ might increase, resulting in less statistical power when comparing conditions to the baseline. However, as shown in Fig. 2, the confidence interval for the model parameters was very similar for the three conditions. In addition, the confidence interval was also very similar for the two sexes. This means that the different contrasts tested have similar statistical power. Considering the significant sexual dichotomy in the processing of music versus noise and the results when comparing either the music or noise with the baseline condition, we conclude that males and females differ in the processing of noise. Thus our data demonstrate a sexual dichotomy in auditory processing. But which mechanism could explain this sexual dichotomy? A key to answering this question is the role of the prefrontal cortex, which we found to be deactivated in men when listening to noise. In humans, the prefrontal cortex is engaged in diverse cognitive processes including cognitive control, working memory, and attention [35]. For example, Gisselgård et al. [36, 37] investigated the influence of irrelevant speech on working memory tasks and revealed a functional link between auditory and prefrontal regions. Tzourio et al. demonstrated that prefrontal areas are engaged in auditory tasks that involve sustained or selective auditory attention [38]. In the present study, no explicit (attention) task was implemented. Subjects lay passively in the scanner and were instructed to listen to the auditory stimuli. While listening to an insignificant stimulus like noise, males deactivated the prefrontal attention areas as compared to silence. Females, on the other hand, had no deactivation of the attention areas and had a higher activation in the primary auditory cortex. Deactivation of the prefrontal regions was only seen in the right hemi-

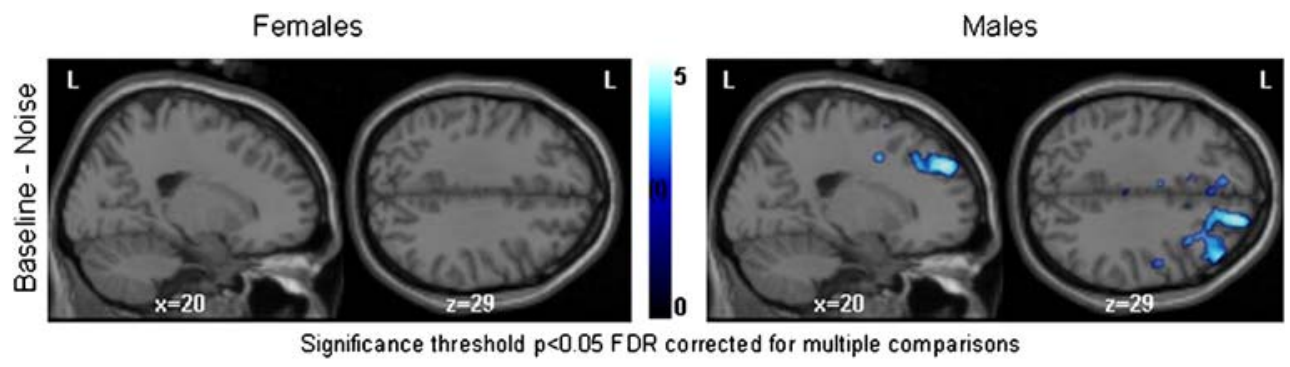

Fig. 4 Spatial distribution of significant decreases in brain activation in men and women for the comparison of noise to the baseline. Clusters are significant at $p<0.05$, FDR corrected for multiple comparisons. $x=20$ means a sagittal plane $20 \mathrm{~mm}$ on the right of the anterior commissure, $z=29$ means a horizontal plane $29 \mathrm{~mm}$ dorsal to the anterior commissure. Only men showed a significant deactivation in the dorsolateral prefrontal cortex 
sphere, which is consistent with Tzourio et al. who stated that a right hemisphere dominance exists for attention [38]. The present results suggest a relationship between activation of PAC and prefrontal cortex. From literature, anatomical evidence exists concerning auditory-prefrontal connections. Studies on monkeys have shown that the prefrontal cortex is reciprocally connected with auditory association areas (i.e. belt and parabelt in the monkey brain) [39]. The secondary and primary auditory areas are reciprocally connected (c.f. [40]). More recent studies on monkeys identified two auditory-prefrontal processing streams: dorso and ventrolateral auditory streams [41-43]. Although, one should be cautious when comparing human and nonhuman primate brains, a similar organization of several parts of the human and monkey prefrontal cortex has been reported [44, 45].

To summarize, we know from literature that the auditory and prefrontal regions are anatomically and functionally linked, and our data show a sexual dichotomy in the (de) activation of both regions. Apparently, the male and female brains handle an insignificant stimulus like noise differently, and we speculate that this is done by a different engagement of the auditory-prefrontal attention network. Namely, differences in attention result in a different deactivation of the right prefrontal cortex, which in turn modulates the activation of the PAC and thus explains the sex differences found in the activation of the PAC. This corresponds with previous findings that sex differences exist in the frontal-temporal network, namely, males have higher intrahemispheric functional connectivity of frontal and temporal areas than females [46]. It is also known from ERP studies that sex differences exist in orienting attention to auditory stimuli [47]. It must be noted that even though our data indicate differences in the auditory system, no independent behavioural data regarding the attention levels during scanning are available. Hence, the present experimental design only allows us to speculate about the correlation between the different deactivation of the prefrontal cortex and differences in attention. Further research is needed to fully clarify the role of attention on PAC activation and to determine whether our results can be repeated. Furthermore, other auditory conditions like pure tones, noise bursts, and speech (sense and nonsense) sounds are needed to fully map the influence of sex on central auditory processing and to investigate lateralization differences between the sexes.

To our knowledge, this is the first time that a sexual dichotomy in the function of the PAC is demonstrated using nonspeech sounds. Previously, a sex difference in the PAC was demonstrated while lip reading $[29,48]$. These studies showed only female activation in the PAC during lip reading because they associate the absent speech sound with the visual lip movements. Males on the other hand, did not display activation in the PAC and focus on the present visual lip image itself. The current study shows that even simple sounds induce different activation patterns, especially in the PAC. Other sexual dichotomies in the central auditory system have been reported. For example, sex differences in auditory feedback loops of the corticofugal network have been found in which men showed more suppression of repeated acoustic stimuli than females. This sex difference in auditory gating is probably the result of differential neuronal inhibition to repeated stimulation [49]. Likewise, the stronger spontaneous otoacoustic emissions (SOAEs) in females are thought to originate from a relatively larger amount of efferent inhibition in males [50]. This efferent innervation would start in the olivary complex and terminate in the outer hair cells of the cochlea [50].

Although the present sexual dichotomy would be consistent with the concept of evolutionary advantages in a hunter-gatherer society (c.f. [51]) where the inhibition of constant irrelevant stimuli in men may facilitate them to focus their attention to a single task, e.g. hunting, this concept remains speculation.

In conclusion, a very significant sexual dichotomy was found in the activation of the PAC with different types of acoustic stimuli (noise and music) together with sex differences in deactivation of prefrontal areas. It is known that the auditory and prefrontal regions are anatomically and functionally linked, and the prefrontal cortex is engaged in auditory attention tasks. Hence, we hypothesize that differences in attention might result in a different involvement of the right prefrontal cortex, which in turn modulates the activation of the PAC. This shows that sex influences brain activity already at the level of primary sensory cortex and that in functional imaging studies on primary sensory cortical areas, sex cannot be ignored.

\section{References}

1. Dekaban AS, Sadowsky D. Changes in brain weights during span of human life: relation of brain weights to body heights and body weights. Ann Neurol 1978;4 4:345-56.

2. Lynn R. Sex differences in intelligence and brain size: a paradox resolved. Pers Individ Differ 1994;17 2:257-71.

3. Peters M, Jancke L, Staiger JF, Schlaug G, Huang Y, Steinmetz H. Unsolved problems in comparing brain sizes in homo sapiens. Brain Cogn 1998;37 2:254-85.

4. Amunts K, Jancke L, Mohlberg H, Steinmetz H, Zilles K. Interhemispheric asymmetry of the human motor cortex related to handedness and gender. Neuropsychologia 2000;38 3:304-12.

5. Good CD, Johnsrude I, Ashburner J, Henson RN, Friston KJ, Frackowiak RS. Cerebral asymmetry and the effects of sex and handedness on brain structure: a voxel-based morphometric analysis of 465 normal adult human brains. Neuroimage 2001;14 3:685-700. 
6. Kovalev VA, Kruggel F, von Cramon DY. Gender and age effects in structural brain asymmetry as measured by MRI texture analysis. Neuroimage 2003;19 3:895-905.

7. Kulynych JJ, Vladar K, Jones DW, Weinberger DR. Gender differences in the normal lateralization of the supratemporal cortex: MRI surface-rendering morphometry of Heschl's gyrus and the planum temporale. Cereb Cortex 1994;4 2:107-18.

8. Hiscock M, Inch R, Jacek C, Hiscock-Kalil C, Kalil KM. Is there a sex difference in human laterality? I. An exhaustive survey of auditory laterality studies from six neuropsychology journals. J Clin Exp Neuropsychol 1994;16 3:423-35.

9. Hiscock M, Israelian M, Inch R, Jacek C, Hiscock-Kalil C. Is there a sex difference in human laterality? II. An exhaustive survey of visual laterality studies from six neuropsychology journals. J Clin Exp Neuropsychol 1995;17 4:590-610.

10. Kansaku K, Kitazawa S. Imaging studies on sex differences in the lateralization of language. Neurosci Res 2001;41 4:333-7.

11. Mcglone J. Sex-differences in human-brain asymmetry. A critical survey. Behav Brain Sci 1980;3 2:215-27.

12. Wisniewski AB. Sexually-dimorphic patterns of cortical asymmetry, and the role for sex steroid hormones in determining cortical patterns of lateralization. Psychoneuroendocrinology 1998;23 5:519-47.

13. Brodmann K. Vergleichende lokalisationlehre der grosshirnrinde. Leipzig: Barth; 1909.

14. Ruytjens L, Willemsen AT, Van Dijk P, Wit HP, Albers FW. Functional imaging of the central auditory system using PET. Acta Otolaryngol 2006;126 12:1236-44.

15. Rademacher J, Morosan P, Schleicher A, Freund HJ, Zilles K. Human primary auditory cortex in women and men. Neuroreport 2001;12 8:1561-5.

16. Harasty J, Double KL, Halliday GM, Kril JJ, McRitchie DA. Language-associated cortical regions are proportionally larger in the female brain. Arch Neurol 1997;54 2:171-6.

17. Leonard CM, Puranik C, Kuldau JM, Lombardino LJ. Normal variation in the frequency and location of human auditory cortex landmarks. Heschl's gyrus: where is it? Cereb Cortex 1998;8 5:397-406.

18. Morosan P, Rademacher J, Schleicher A, Amunts K, Schormann T, Zilles K. Human primary auditory cortex: cytoarchitectonic subdivisions and mapping into a spatial reference system. Neuroimage 2001;13 4:684-701.

19. Penhune VB, Zatorre RJ, MacDonald JD, Evans AC. Interhemispheric anatomical differences in human primary auditory cortex: probabilistic mapping and volume measurement from magnetic resonance scans. Cereb Cortex 1996;6 5:661-72.

20. Rademacher J, Morosan P, Schormann T, Schleicher A, Werner C, Freund HJ, et al. Probabilistic mapping and volume measurement of human primary auditory cortex. Neuroimage 2001;13 4:669-83.

21. Rademacher J, Burgel U, Zilles K. Stereotaxic localization, intersubject variability, and interhemispheric differences of the human auditory thalamocortical system. Neuroimage 2002;17 $1: 142-60$.

22. Kansaku K, Yamaura A, Kitazawa S. Sex differences in lateralization revealed in the posterior language areas. Cereb Cortex 2000;10 9:866-72.

23. Obleser J, Eulitz C, Lahiri A, Elbert T. Gender differences in functional hemispheric asymmetry during processing of vowels as reflected by the human brain magnetic response. Neurosci Lett 2001;314 3:131-4.

24. Kaiser A, Kuenzli E, Zappatore D, Nitsch C. On females' lateral and males' bilateral activation during language production: A fMRI study. Int J Psychophysiol 2007;63 2:192-8.

25. Frost JA, Binder JR, Springer JA, Hammeke TA, Bellgowan PS, Rao SM, et al. Language processing is strongly left lateralized in both sexes. Evidence from functional MRI. Brain 1999;122 Pt 2:199-208.
26. Sommer IE, Aleman A, Bouma A, Kahn RS. Do women really have more bilateral language representation than men? A meta-analysis of functional imaging studies. Brain 2004;127 Pt 8:1845-52.

27. Kocak M, Ulmer JL, Biswal BB, Aralasmak A, Daniels DL, Mark LP. The influence of gender on auditory and language cortical activation patterns: preliminary data. AJNR Am J Neuroradiol 2005;26 9:2248-55.

28. Goldstein JM, Jerram M, Poldrack R, Anagnoson R, Breiter HC, Makris N, et al. Sex differences in prefrontal cortical brain activity during fMRI of auditory verbal working memory. Neuropsychology 2005;19 4:509-19.

29. Ruytjens L, Albers F, van Dijk P, Wit H, Willemsen A. Activation in primary auditory cortex during silent lipreading is determined by sex. Audiol Neurotol (in press).

30. Reinders AA, Willemsen AT, Georgiadis JR, Hovius M, Paans $\mathrm{AM}$, den Boer JA. Interscan displacement-induced variance in PET activation data is excluded by a scan-specific attenuation correction. Neuroimage 2002;17 4:1844-53.

31. Friston KJ, Ashburner J, Frith CD, Poline JB, Heather JD, Frackowiak RSJ. Spatial registration and normalization of images. Hum Brain Mapp 1995;3 3:165-89.

32. Genovese CR, Lazar NA, Nichols T. Thresholding of statistical maps in functional neuroimaging using the false discovery rate. Neuroimage 2002;15 4:870-8.

33. Eickhoff SB, Stephan KE, Mohlberg H, Grefkes C, Fink GR, Amunts K, et al. A new SPM toolbox for combining probabilistic cytoarchitectonic maps and functional imaging data. Neuroimage 2005;25 4:1325-35.

34. Matthew Brett, Anton J-L, Valabregue R, Poline J-B. Region of interest analysis using an SPM toolbox [abstract]. Presented at the 8th International Conference on Functional Mapping of the Human Brain, 2-6 June 2002, Sendai, Japan.

35. Miller EK, Cohen JD. An integrative theory of prefrontal cortex function. Annu Rev Neurosci 2001;24:167-202.

36. Gisselgard J, Petersson KM, Baddeley A, Ingvar M. The irrelevant speech effect: A PET study. Neuropsychologia 2003;41 14:1899-911.

37. Gisselgard J, Petersson KM, Ingvar M. The irrelevant speech effect and working memory load. Neuroimage 2004;22 3:1107-16.

38. Tzourio N, Massioui FE, Crivello F, Joliot M, Renault B, Mazoyer B. Functional anatomy of human auditory attention studied with PET. Neuroimage 1997;5 1:63-77.

39. Barbas H, Mesulam MM. Cortical afferent input to the principalis region of the rhesus monkey. Neuroscience $1985 ; 15$ 3:619-37.

40. Kaas JH, Hackett TA. Subdivisions of auditory cortex and levels of processing in primates. Audiol Neurootol 1998;3 2-3:73-85.

41. Romanski LM, Tian B, Fritz J, Mishkin M, Goldman-Rakic PS, Rauschecker JP. Dual streams of auditory afferents target multiple domains in the primate prefrontal cortex. Nat Neurosci 1999;2 12:1131-6.

42. Romanski LM, Bates JF, Goldman-Rakic PS. Auditory belt and parabelt projections to the prefrontal cortex in the rhesus monkey. J Comp Neurol 1999;403 2:141-57.

43. Romanski LM, Goldman-Rakic PS. An auditory domain in primate prefrontal cortex. Nat Neurosci 2002;5 1:15-6.

44. Ongur D, Ferry AT, Price JL. Architectonic subdivision of the human orbital and medial prefrontal cortex. J Comp Neurol 2003;460 3:425-49.

45. Petrides M, Pandya DN. Comparative cytoarchitectonic analysis of the human and the macaque ventrolateral prefrontal cortex and corticocortical connection patterns in the monkey. Eur J Neurosci 2002;16 2:291-310

46. Gootjes L, Bouma A, Van Strien JW, Scheltens P, Stam CJ. Attention modulates hemispheric differences in functional connectivity: evidence from MEG recordings. Neuroimage 2006;30 1:245-53. 
47. Nagy E, Potts GF, Loveland KA. Sex-related ERP differences in deviance detection. Int J Psychophysiol 2003;48 3:285-92.

48. Ruytjens L, Albers F, van Dijk P, Wit H, Willemsen A. Neural responses to silent lipreading in normal hearing male and female subjects. Eur J Neurosci 2006;24 6:1835-44.

49. Hetrick WP, Sandman CA, Bunney WE Jr, Jin Y, Potkin SG, White $\mathrm{MH}$. Gender differences in gating of the auditory evoked potential in normal subjects. Biol Psychiatry 1996;39 1:51-8.
50. McFadden D. A speculation about the parallel ear asymmetries and sex differences in hearing sensitivity and otoacoustic emissions. Hear Res 1993;68 2:143-51.

51. Kimura D. Sex and cognition. London: MIT Press; 1999.

52. Mai JK, Assheuer J, Paxinos G. Atlas of the human brain. San Diego: Academic; 1997.

53. Talairach J, Tournoux P. Co-planar atlas of the human brain. New York: Springer; 1988. 